\title{
TURÁN'S PURE POWER SUM PROBLEM
}

\author{
A. Y. CHEER AND D. A. GOLDSTON
}

\begin{abstract}
Let $1=z_{1} \geq\left|z_{2}\right| \geq \cdots \geq\left|z_{n}\right|$ be $n$ complex numbers, and consider the power sums $s_{\nu}=z_{1}{ }^{\nu}+z_{2}{ }^{2}+\cdots+z_{n}{ }^{\nu}, 1 \leq \nu \leq n$. Put $R_{n}=$ $\min \max _{1 \leq \nu \leq n}\left|s_{\nu}\right|$, where the minimum is over all possible complex numbers satisfying the above. Turán conjectured that $R_{n}>A$, for $A$ some positive absolute constant. Atkinson proved this conjecture by showing $R_{n}>1 / 6$. It is now known that $1 / 2<R_{n}<1$, for $n \geq 2$. Determining whether $R_{n} \rightarrow 1$ or approaches some other limiting value as $n \rightarrow \infty$ is still an open problem. Our calculations show that an upper bound for $R_{n}$ decreases for $n \leq 55$, suggesting that $R_{n}$ decreases to a limiting value less than 0.7 as $n \rightarrow \infty$.
\end{abstract}

\section{INTRODUCTION}

Let $z_{1}, z_{2}, \ldots, z_{n}$ be complex numbers and let

$$
s_{\nu}=\sum_{k=1}^{n} z_{k}^{\nu}
$$

be the $\nu$ th (pure) power sum of these $n$ complex numbers. Turán initiated the study of the problem of determining how small $\left|s_{\nu}\right|$ can be for all $\nu$ in some range and $z_{k}$ satisfying various conditions. Depending on the conditions imposed on the range of values of $\nu$ and $z_{k}$, and also on the norm used in obtaining the lower bound, many interesting problems arise, see [8, Chapters 1-4]. We are concerned with the problem of determining how small $\left|s_{\nu}\right|$ can be for all $1 \leq \nu \leq m$ and for any configuration of complex numbers satisfying

$$
1=z_{1} \geq\left|z_{2}\right| \geq \cdots \geq\left|z_{n}\right| .
$$

This condition on the $z_{k}$ 's is not particularly restrictive since many more general problems can quickly be reduced to this case. We let

$$
M_{n}(m)=\max _{1 \leq \nu \leq m}\left|s_{\nu}\right|, \quad R_{n}(m)=\min _{1=z_{1} \geq\left|z_{2}\right| \geq \cdots \geq\left|z_{n}\right|} M_{n}(m)
$$

i.e., $R_{n}(m)$ is the min-max of the absolute value of the first $m$ power sums of $n$ complex numbers satisfying (2). Since the maximum is nondecreasing with $m$, we

Received by the editor March 4, 1995.

1991 Mathematics Subject Classification. Primary 11N30.

Key words and phrases. Turán's method.

Research of the first author was supported in part by the Institute for Theoretical Dynamics, University of California at Davis.

Research of the second author was supported in part by NSF Grant DMS9205533 and NSF Computing Research Environments Award 9303986.

(C)1996 American Mathematical Society 
see that $R_{n}(m)$ is nondecreasing in $m$. Taking $z_{k}=e(k / n)$, for $1 \leq k \leq n$, where $e(u)=e^{2 \pi i u}$, we see that $s_{\nu}=0$ for $1 \leq \nu \leq n-1$. Therefore,

$$
R_{n}(m)=0 \quad \text { for } 1 \leq m \leq n-1 .
$$

The first case of interest is thus when $m=n$, the min-max of $n$ complex numbers over the first $n$ power sums. For this case, denote

$$
M_{n}=M_{n}(n), \quad R_{n}=R_{n}(n) .
$$

Turán conjectured in 1938 that $R_{n}$ is bounded below by an absolute constant for all $n$. This conjecture was proved by Atkinson [1] in 1961, where he obtained

$$
R_{n}>1 / 6 .
$$

Atkinson subsequently improved his result to $R_{n}>1 / 3$ and also obtained a somewhat larger lower bound for some $n$ [2]. Very recently, Biró [3] found an elegant elementary argument which shows

$$
R_{n}>1 / 2 .
$$

On the other hand, Komlós, Sárközy, and Szemerédi [4] showed that

$$
R_{n}<1-\frac{1}{250 n}
$$

for all sufficiently large $n$. In view of these results the main question to be examined is whether $R_{n} \rightarrow 1$ as $n \rightarrow \infty$ or whether it decreases to some limiting value. Both possibilities have been suggested, and it appears Turán himself has at various times conjectured both possibilities [8, p. 30], [9, p. XVII]. The only known values are $R_{1}=1, R_{2}=0.87403 \ldots$, and $R_{3}=0.8247830 \ldots$. Here $R_{1}=1$ is trivial, and $R_{2}=\sqrt{3-\sqrt{5}}$ may be obtained by a relatively easy Lagrange multiplier argument. The value $\left(R_{2}\right)^{2}$ satisfies the equation

$$
x^{2}-6 x+4=0 .
$$

Determination of $R_{3}$ is much harder. Lawrynowicz [6] determined $R_{3}$ algebraically in 1960, and obtained the numerical value given above in 1967 [7]. The value $\left(R_{3}\right)^{2}$ satisfies the equation ${ }^{1}$

$$
\begin{aligned}
& x^{15}-81 x^{14}+2613 x^{13}-43629 x^{12}+417429 x^{11}-2450985 x^{10}+9516137 x^{9} \\
& -26203659 x^{8}+53016480 x^{7}-83714418 x^{6}+112601340 x^{5}-140002992 x^{4} \\
& +156204288 x^{3}-124361568 x^{2}+55427328 x-10077696=0 .
\end{aligned}
$$

In this paper we report on some computations which extend our knowledge of $R_{n}$ and suggest that $R_{n}$ decreases to a limiting value. Our results are summarized in the following theorem.

\footnotetext{
${ }^{1}$ Ławrynowicz determined $\left(R_{3}\right)^{2}$ in terms of a different algebraic number which satisfied an equation of degree 15. The equation above was obtained for us by David Bailey, who used the Ferguson-Forcade algorithm and his multiprecision arithmetic package for finding integer relations between numbers.
} 
TABLE 1

\begin{tabular}{|c|c||c|c|}
\hline$n$ & $M_{n}$ & $n$ & $M_{n}$ \\
\hline \hline 2 & 0.87403204889764214159 & 27 & 0.71351379274002663079 \\
3 & 0.82478303091462249657 & 28 & 0.71280297643602584513 \\
4 & 0.79787183891219785566 & 29 & 0.71213492854485684518 \\
5 & 0.78068751175852318911 & 30 & 0.71150571445562842882 \\
6 & 0.76866240212865063009 & 31 & 0.71091187832481901227 \\
7 & 0.75972275127528535640 & 32 & 0.71035037153531655407 \\
8 & 0.75278476350214275366 & 33 & 0.70981849370598932067 \\
9 & 0.74722423731338641938 & 34 & 0.70931384374342863335 \\
10 & 0.74265504914476718905 & 35 & 0.70883427898651897355 \\
11 & 0.73882487313790486797 & 36 & 0.70837788091679418294 \\
12 & 0.73556147974443498507 & 37 & 0.70794292622936772579 \\
13 & 0.73274307211717818350 & 38 & 0.70752786230650853148 \\
14 & 0.73028094927147070166 & 39 & 0.70713128632741405830 \\
15 & 0.72810889170059241298 & 40 & 0.70675192739708693051 \\
16 & 0.72617640456896152304 & 41 & 0.70638863119452338965 \\
17 & 0.72444427147342536314 & 42 & 0.70604034673315181320 \\
18 & 0.72288154379177815772 & 43 & 0.70570611490021873219 \\
19 & 0.72146345068685877134 & 44 & 0.70538505850083375768 \\
20 & 0.72016991610628663886 & 45 & 0.70507637357986472730 \\
21 & 0.71898448586498316988 & 46 & 0.70477932183327719645 \\
22 & 0.71789353784931532652 & 47 & 0.70449322395173041892 \\
23 & 0.71688569151654277288 & 48 & 0.70421745376474166007 \\
24 & 0.71595136015246079880 & 49 & 0.70395143307465529630 \\
25 & 0.71508240701664073135 & 50 & 0.70369462708689897930 \\
26 & 0.71427187818188997713 & 55 & 0.70253266322361406713 \\
\hline
\end{tabular}

Theorem. There exist configurations of points satisfying equation (2) with $M_{n}$ given in Table 1. These numbers are accurate to 20 digits.

\section{The Computational SChEme}

Our method for computing $R_{n}$ is due to Lawrynowicz [6]. He proved that among the extremal configurations of points $z_{k}$ which takes on the value $R_{n}$, there is always a configuration with

$$
\left|s_{1}\right|=\left|s_{2}\right|=\cdots=\left|s_{n}\right| .
$$

This is possibly the only type of configuration where $R_{n}$ is attained, but this is not known. Consequently, we can formulate the computing of $R_{n}$ as a Lagrange multiplier problem. To compute $R_{n}$, let $z_{1}=1, z_{k}=x_{k}+i y_{k}$ for $2 \leq k \leq n$, and

$$
\begin{aligned}
& F\left(x_{2}, x_{3}, \ldots, x_{n}, y_{2}, \ldots, y_{n}, \lambda_{1}, \lambda_{2}, \ldots, \lambda_{n-1}\right) \\
& =\left|s_{1}\right|^{2}-\sum_{k=1}^{n-1} \lambda_{k}\left(\left|s_{1}\right|^{2}-\left|s_{k+1}\right|^{2}\right) .
\end{aligned}
$$


Then the equation $R_{n}=\left|s_{1}\right|$ is satisfied at a critical point of $F$. To obtain this critical point, we need to solve the system of $3 n-3$ equations

$$
\frac{\partial F}{\partial x_{j+1}}=0, \quad \frac{\partial F}{\partial y_{j+1}}=0, \quad \frac{\partial F}{\partial \lambda_{j}}=0, \quad \text { for } j=1,2, \ldots, n-1 .
$$

Each equation is a polynomial in $3 n-3$ variables of degree $\leq 2 n$ in each variable. For example, when $n=2$ we have $z_{1}=1, z_{2}=x_{2}+i y_{2},\left|s_{1}\right|^{2}=\left(1+x_{2}\right)^{2}+y_{2}^{2}$, $\left|s_{2}\right|^{2}=\left(1+x_{2}{ }^{2}-y_{2}{ }^{2}\right)^{2}+4 x_{2}^{2} y_{2}^{2}$, and our system is

$$
\begin{aligned}
& \text { (11a) } \frac{\partial F}{\partial x_{2}}=2\left(1+x_{2}\right)-\lambda_{1}\left(2\left(1+x_{2}\right)-4 x_{2}\left(1+x_{2}^{2}-y_{2}^{2}\right)-8 x_{2} y_{2}^{2}\right)=0 \\
& \text { (11b) } \frac{\partial F}{\partial y_{2}}=2 y_{2}-\lambda_{1}\left(2 y_{2}+4 y_{2}\left(1+x_{2}^{2}-y_{2}^{2}\right)-8 x_{2}^{2} y_{2}\right)=0 \\
& \text { (11c) } \frac{\partial F}{\partial \lambda_{1}}=\left(1+x_{2}\right)^{2}+y_{2}^{2}-\left(1+x_{2}^{2}-y_{2}^{2}\right)^{2}-4 x_{2}^{2} y_{2}^{2}=0
\end{aligned}
$$

This system may be solved by hand; equations (11a) and (11b) imply $1+2 x_{2}=$ $x_{2}{ }^{2}+y_{2}{ }^{2}$. From equation (11c) we get $x_{2}=(1 \pm \sqrt{5}) / 4$, and $y_{2}= \pm \sqrt{3} x_{2}$. The value $y_{2}=1+\sqrt{5}$ is outside the unit circle. Therefore the value for $R_{2}$ is $\sqrt{3-\sqrt{5}}$, which is obtained when $x_{2}=(1-\sqrt{5}) / 4$, and $y_{2}= \pm \sqrt{3} x_{2}$.

This example illustrates one of the procedures we use to compute $R_{n}$. To find the critical points of (10), Newton's method is used. At each critical point we compute $\left|s_{1}\right|$; the smallest such value is $R_{n}$. As will be described below, we cannot actually carry this procedure out completely. What we actually compute is a configuration which is likely to give the value $R_{n}$, and this configuration of points, independent of the computational scheme, can be checked to give a small value for $M_{n}$, and thus provides a rigorously proven upper bound for $R_{n}$.

\section{THE COMPUTATION}

All of our computations make use of Mathematica. The system of equations (10) is solved numerically using a Newton iterative scheme which converges quadratically to the solution that is closest to the given initial guess. To obtain all solutions of (10) with $M_{n}<1$, random initial guesses are used in Newton's method. The program is run with thousands of different random initial guesses and continued until no further new critical points are obtained in over several thousand runs. For $n=3$, only two critical points are found, one of which gives Eawrynowicz's solution, and the other is outside the unit circle with the value $1.374 \ldots$. Proceeding in this fashion, we compute the critical points for $n=4,5,6$. The resulting smallest values for $M_{n}$ at the critical points are listed in Table 2. By the time $n=6,56$ critical points with $M_{n}<1$ and 125 critical points with $M_{n}<1.45$ are found. This procedure does not ensure that the values in Table 2 are a complete list of all critical points. At $n=7$, the scheme of using random initial guesses in Newton's algorithm for solving (10) is infeasible for finding $R_{n}$. First, the Newton algorithm will only converge infrequently, and second, there are large numbers of critical points. This algorithm, for $n=7$, took several months of CPU time before yielding the value given in Table 1. 
TABLE 2

Values of $M_{n}<1$ at critical points (not all 56 values found for $n=6$ shown)

\begin{tabular}{|c|c|c|c|}
\hline$n=3$ & $n=4$ & $n=5$ & $n=6$ \\
\hline 0.8247830309 & $\begin{array}{l}0.7978718389 \\
0.8389236336 \\
0.8762366068 \\
0.9050025366 \\
0.9074678426 \\
0.9504184932 \\
0.9577375152 \\
0.9746757164 \\
0.9885588908\end{array}$ & $\begin{array}{l}0.7806875117 \\
0.8337671859 \\
0.8690990094 \\
0.8712303154 \\
0.8727150203 \\
0.8825304010 \\
0.8829360128 \\
0.8829360128 \\
0.9069016543 \\
0.9333716319 \\
0.9413655298 \\
0.9443800869 \\
0.9550142456 \\
0.9677037216 \\
0.9828023416 \\
0.9889082339 \\
0.9922111204\end{array}$ & $\begin{array}{r}0.7686624021 \\
0.8111179657 \\
0.8285874602 \\
0.8393034012 \\
0.8432191421 \\
0.8483174001 \\
0.8503894070 \\
0.8537885153 \\
0.8556601896 \\
0.8613214008 \\
0.8737623150 \\
0.8740381632 \\
0.8894480590 \\
0.8958343035 \\
0.8958688134 \\
0.8972435100 \\
0.9178211517 \\
0.9202763035 \\
\ldots \\
0.9996198736 \\
0.9996426733 \\
0.9998003871 \\
0.9998070146\end{array}$ \\
\hline
\end{tabular}

We can draw two conclusions from these calculations. First, because of the small number of critical points with $M_{4} \leq 1$, it may be possible but very laborious to prove that the upper bound for $R_{4}$ in Table 1 is actually equal to $R_{4}$. Secondly, because the next smallest value of $M_{n}$ found for $n=4,5,6$ is considerably larger than the smallest value, it is very likely that the numbers in Table 1 are the correct values for $R_{4}, R_{5}$, and $R_{6}$.

To go beyond $n=6$ we use information obtained from our extremal configurations for $n=3,4,5$ and 6 . These are shown in Figures 1a and 1b. The power sums display a very regular pattern and they lie on a circle as required by equation (8). In every case we find that they move from $s_{1}$ to $s_{n}$ in order, clockwise, starting from the top position. Further, as $n$ increases the points shift in a regular pattern. By computing difference tables we obtained a guess for the case $n=7$. That is, we obtain $s_{k}, 1 \leq k \leq 7$. One can then obtain the $z$ 's from the $s$ 's by solving the following equations:

$$
p(z)=\prod_{k=1}^{n}\left(z-z_{k}\right)=\sum_{k=0}^{n} a_{k} z^{n-k}, \quad z_{1}=1, \quad a_{0}=1
$$



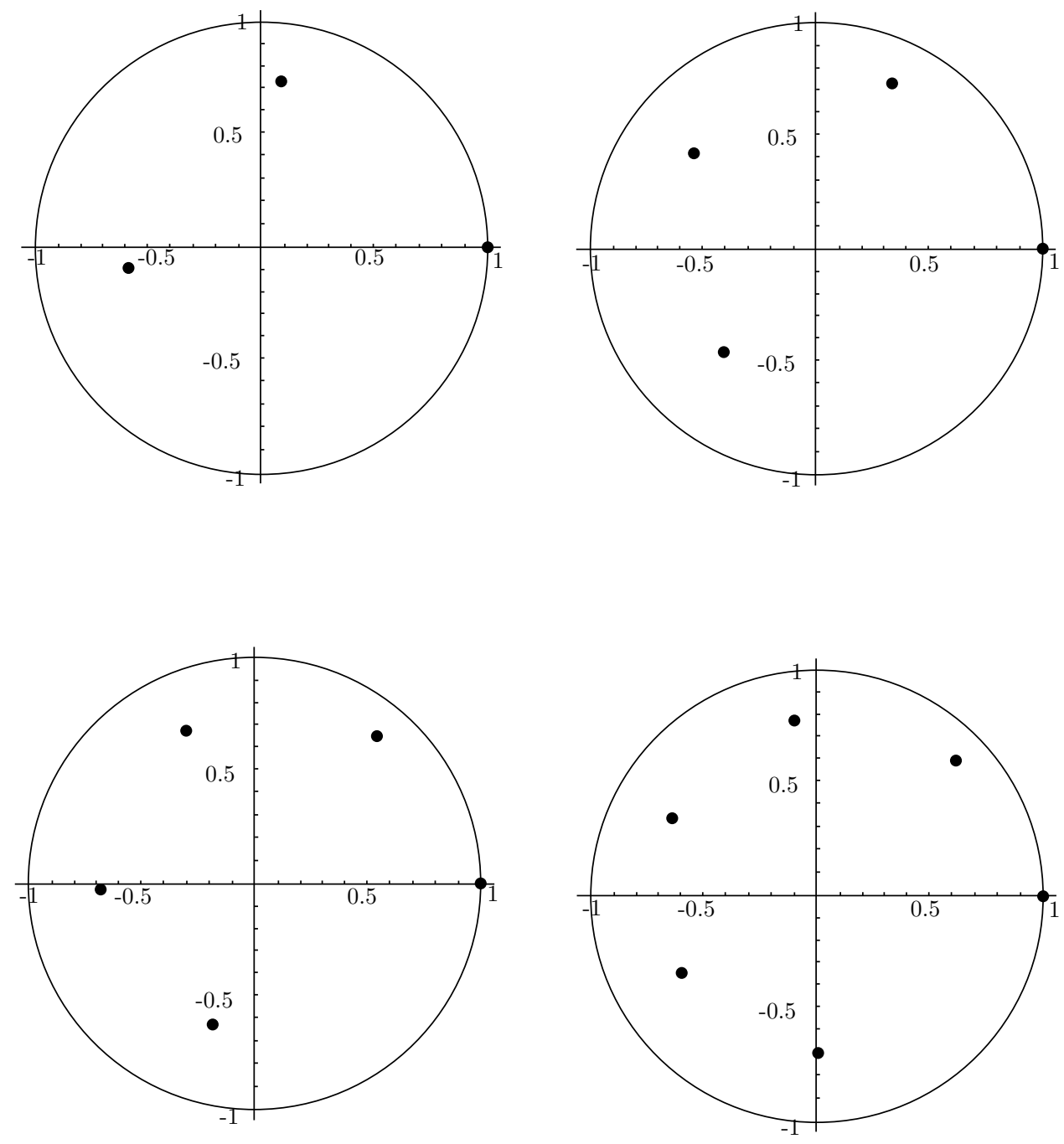

Figure 1a. Extremal configuration of $z_{k}$ 's for $n=3,4,5,6$

and

$$
a_{k}=(-1)^{k} \frac{1}{k !} \operatorname{det}\left(\begin{array}{ccccccc}
s_{1} & 1 & 0 & 0 & 0 & \ldots & 0 \\
s_{2} & s_{1} & 2 & 0 & 0 & \ldots & 0 \\
s_{3} & s_{2} & s_{1} & 3 & 0 & \ldots & 0 \\
\vdots & \vdots & \vdots & \vdots & \ddots & \vdots & \\
s_{k-1} & s_{k-2} & s_{k-3} & s_{k-4} & s_{k-5} & \ldots & k \\
s_{k} & s_{k-1} & s_{k-2} & s_{k-3} & s_{k-4} & \ldots & s_{1}
\end{array}\right)
$$

The $a_{k}$ 's are computed by (13) using the $s$ 's determined by our difference procedure. From (12), $p(z)$ is obtained. Newton's method is then used to find the roots $z_{k}$ of $p(z)$. These $z_{k}$ 's are used as the initial starting guess for the Newton method used 

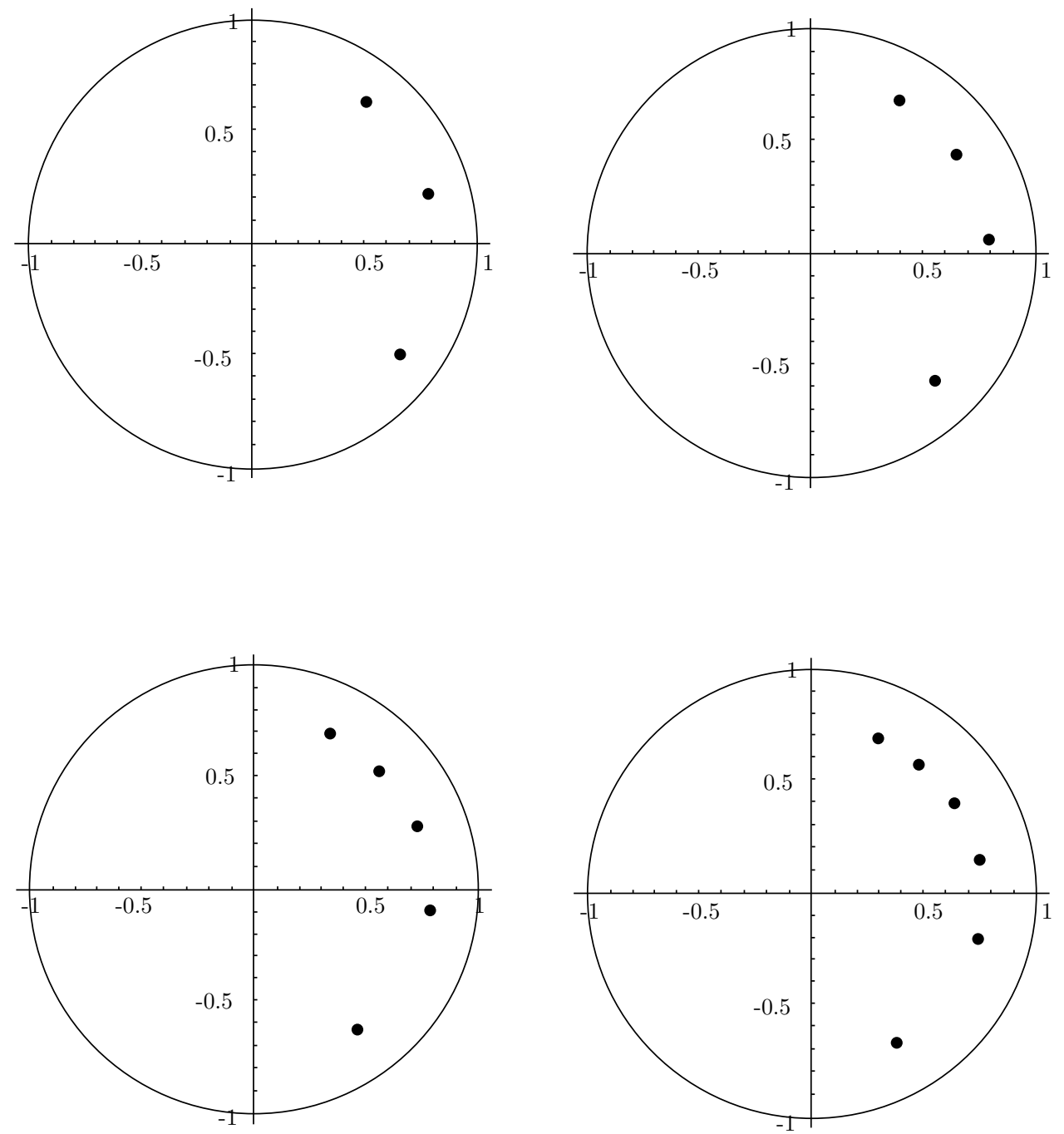

FIgURE 1b. Extremal configuration of $s_{k}$ 's for $n=3,4,5,6$

to solve (10). In practice, we found that Newton's method with this initial guess converged immediately to the answer. This procedure was used to compute Table 1 up to $n=36$. At this point, the Newton method used to obtain the $z$ 's from the $s$ 's in (12) and (13) takes a considerable amount of CPU time. Instead, since the $z$ configuration is now very regular, we use an interpolation routine to guess the new $z$ 's from our previous results directly, bypassing equations (12) and (13). By this point it is also possible to obtain at once guesses for the $z_{k}$ 's not just for the next $n$, but for the next five $n$ with sufficient accuracy. These points are used as initial guesses in the Newton algorithm to solve (10). Using this procedure we compute up to $M_{50}$.

When we compute the power sums directly from our guess of $z_{k}$ 's, without first solving (10), we obtain slightly higher values for $M_{n}$. In particular, using 
the configurations for $n \leq 50$ we obtain $R_{51} \leq 0.70344665, R_{52} \leq 0.7032078$, $R_{53} \leq 0.702982, R_{54} \leq 0.70281$, and $R_{55} \leq 0.702871$. In comparison, for the case of $n=55$, if the configuation obtained above is used as the initial guess to Newton's algorithm to solve (10) the value $R_{55} \leq 0.702532 \ldots$ in Table 1 is obtained. The first 50 configurations give guesses for the $z_{k}$ 's with $M_{n}<1$ for $n \leq 62$.

\section{Conclusions}

From our calculations, it is likely that $R_{n}$ decreases to a limiting value. In Figure 2 we display some of the extremal solutions found by our calculations. The extremal configuration of $z$ 's are converging to a limiting configuration. On the other hand, the distribution of the power sums has not developed sufficiently to predict the ultimate pattern it will take.
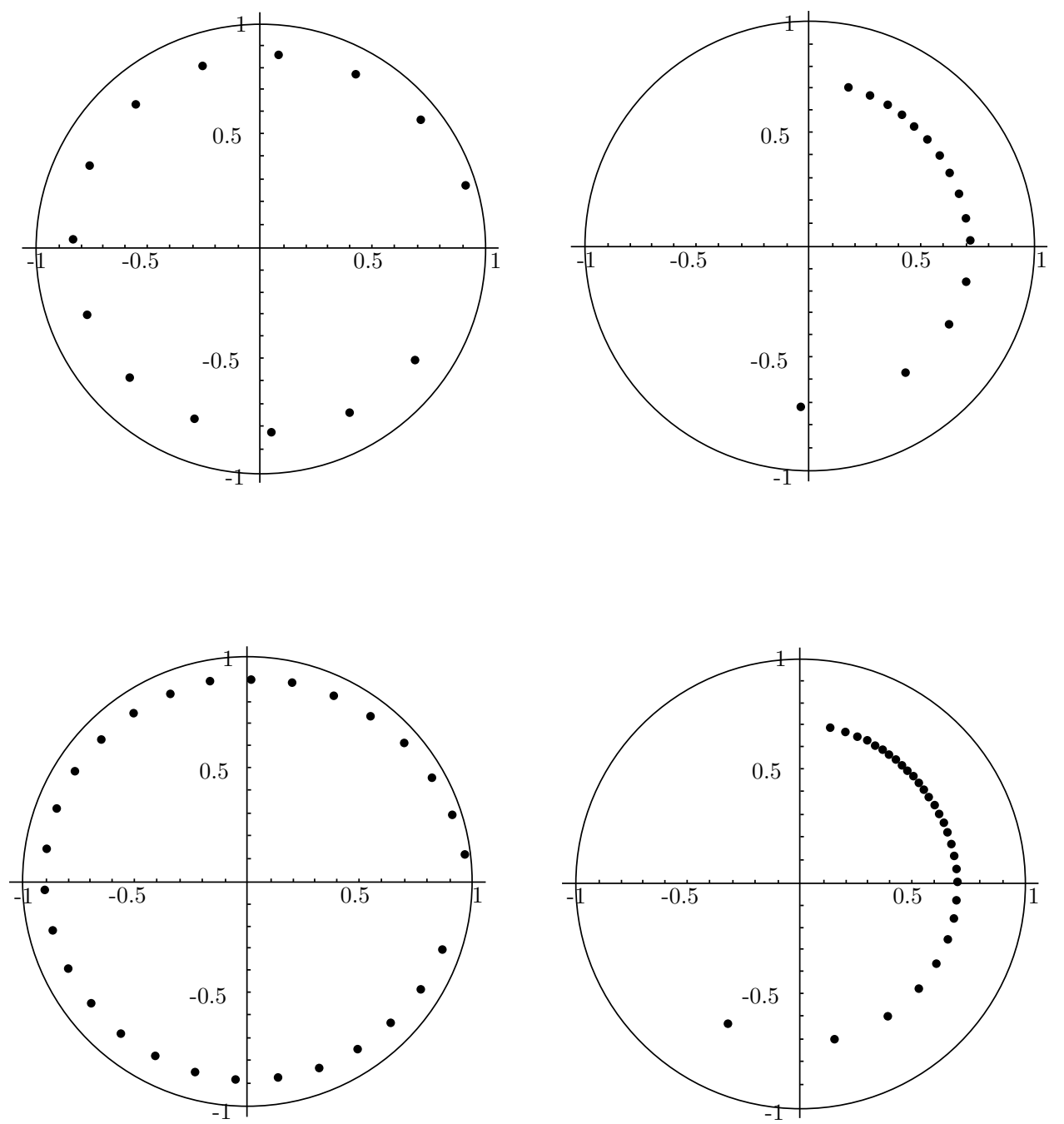

FiguRE 2a. Extremal configuration of $z_{k}$ 's and $s_{k}$ 's for $n=15,30$ 

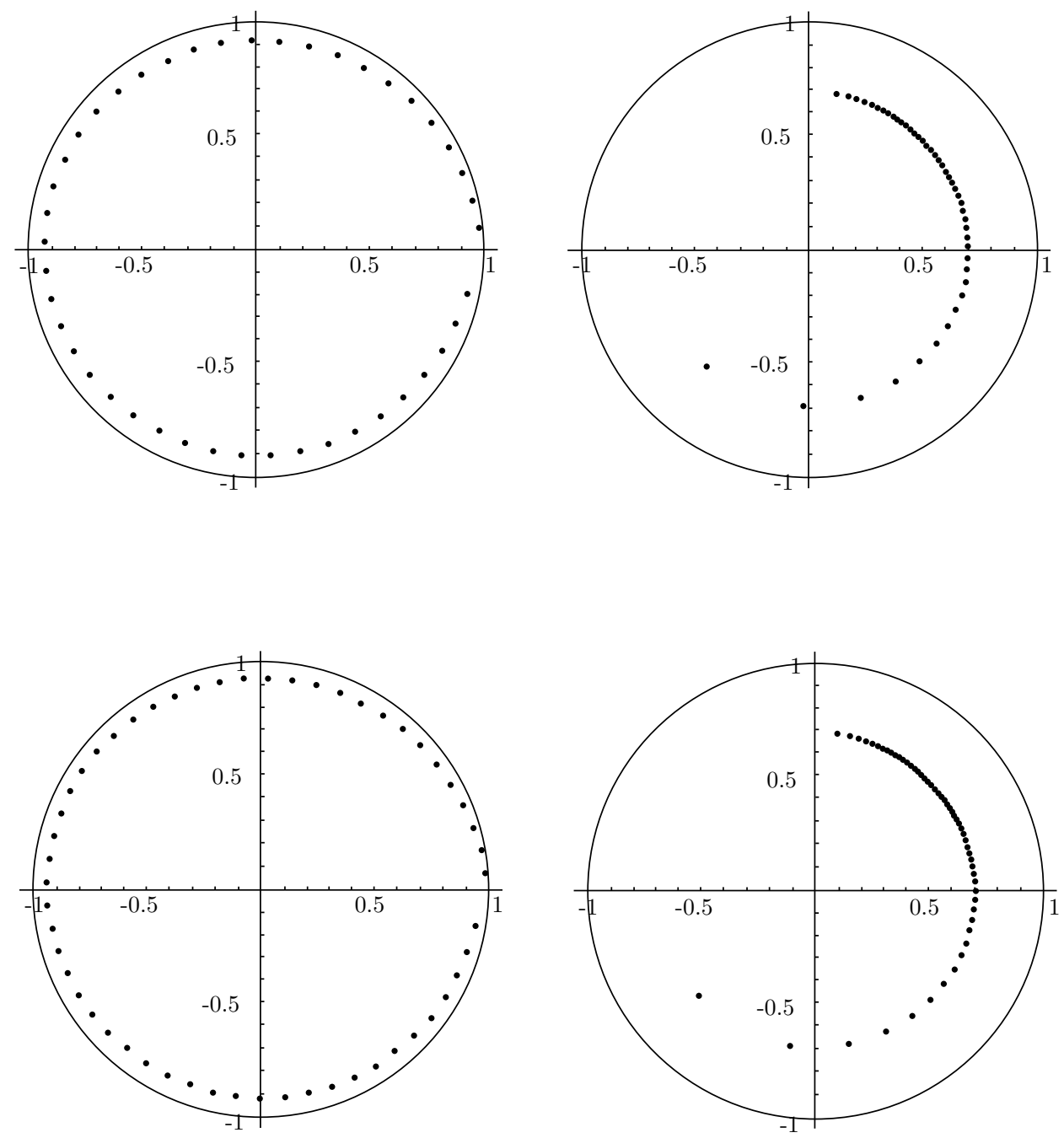

FigURE 2b. Extremal configuration of $z_{k}$ 's and $s_{k}$ 's for $n=45,55$

\section{REFERENCES}

1. F. V. Atkinson, On sums of powers of complex numbers, Acta Math. Acad. Sci. Hung. 12 (1961), 185-188. MR 23:A3714

2. F. V. Atkinson, Some further estimates concerning sums of powers of complex numbers, Acta Math. Acad. Sci. Hung. 20 (1969), 193-210. MR 39:443

3. A. Biró, On a problem of Turán concerning sums of powers of complex numbers, Acta Math. Hungar. 65 (1994), no. 3, 09-216. MR 95d:11120

4. J. Komlós, A. Sárközy, E. Szemerédi, On sums of powers of complex numbers (in Hungarian), Mat. Lapok 15 (1964), 337-347. MR 34:2534

5. J. Ławrynowicz, Remark on a problem of P. Turán, Bull. Soc. Sci. Lettres. Łódź 11 (1960), no. 1, 1-4. MR 23:A3959

6. J. Ławrynowicz, Calculation of a minimum maximorum of complex numbers, Bull. Soc. Sci. Lettres. Łódź, 11 (1960), no. 2, 1-9. MR 23:A3958 
7. J. Ławrynowicz, Remark on power-sums of complex numbers, Acta Math. Hungar. 18 (1967), no. 3-4, 279-281. MR 36:379

8. P. Turán, On a new method of analysis and its applications, John Wiley \& Sons, New York, 1984. MR 86b: 11059

9. P. Turán, Collected Papers of Paul Turán, edited by Paul Erdös, Budapest: Akademiai Kiado, 1990. MR 91i:01145

Department of Mathematics and Institute of Theoretical Dynamics, University of California, Davis, California 95616

E-mail address: goldston@jupiter.sjsu.edu

Department of Mathematics and Computer Science, San Jose State University, San Jose, California 95192

E-mail address: aycheer@ucdavis.edu 